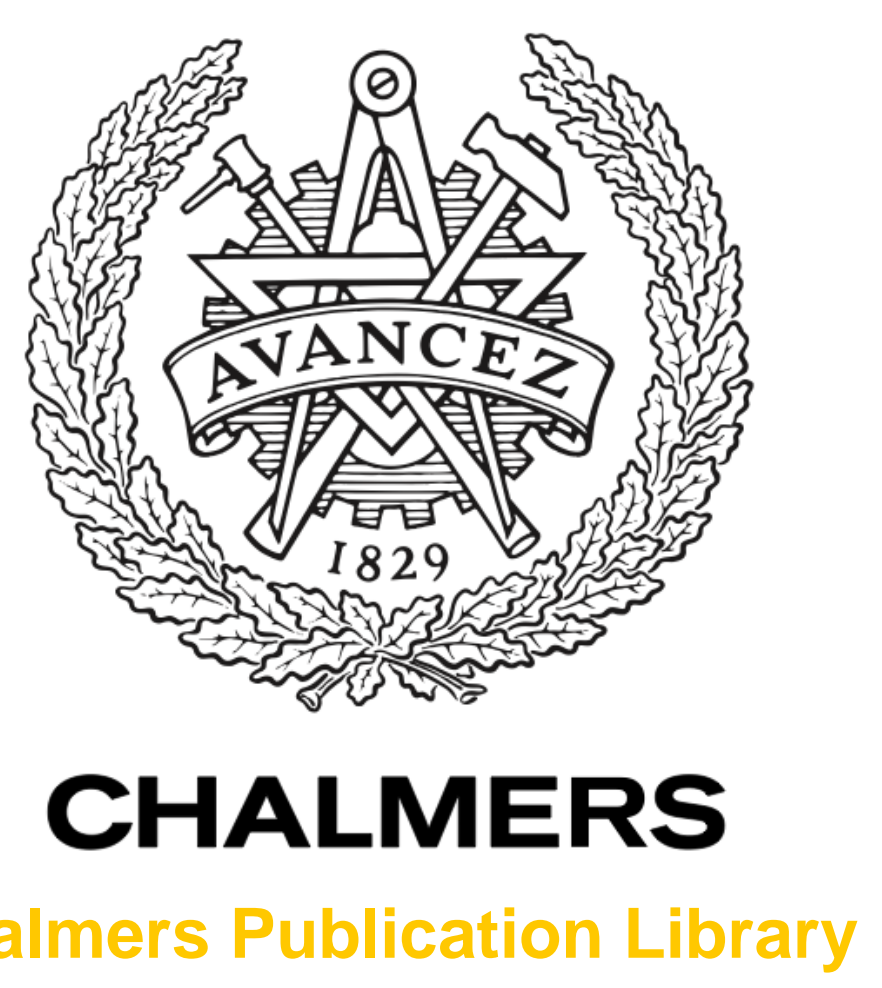

Chalmers Publication Library

\title{
Long-Haul (3465 km) Transmission of a 10 GBd QPSK Signal with Low Noise Phase-Sensitive In-Line Amplification
}

This document has been downloaded from Chalmers Publication Library $(\mathrm{CPL})$. It is the author's version of a work that was accepted for publication in:

\section{European Conference on Optical Communication (ECOC) 2014}

Citation for the published paper:

Olsson, S. ; Lundström, C. ; Karlsson, M. (2014) "Long-Haul (3465 km) Transmission of a 10 GBd QPSK Signal with Low Noise Phase-Sensitive In-Line Amplification". European Conference on Optical Communication (ECOC) 2014 pp. Paper PD.2.2.

http://dx.doi.org/10.1109/ECOC.2014.6964278

Downloaded from: http://publications.lib.chalmers.se/publication/207171

Notice: Changes introduced as a result of publishing processes such as copy-editing and formatting may not be reflected in this document. For a definitive version of this work, please refer to the published source. Please note that access to the published version might require a subscription. 


\title{
Long-Haul (3465 km) Transmission of a 10 GBd QPSK Signal with Low Noise Phase-Sensitive In-Line Amplification
}

\author{
Samuel L.I. Olsson(1), Carl Lundström(1), Magnus Karlsson ${ }^{(1)}$, and Peter A. Andrekson ${ }^{(1)}$ \\ (1) Photonics Laboratory, Department of Microtechnology and Nanoscience, Chalmers University of Tech- \\ nology, SE-412 96, Gothenburg, Sweden, samuel.olsson@chalmers.se
}

\begin{abstract}
We report the first use of low noise in-line phase-sensitive amplifiers in a long-haul circulating loop experiment. A reach extension of $200 \%$ compared to using EDFAs is observed with a $10 \mathrm{GBd}$ QPSK signal.
\end{abstract}

\section{Introduction}

Fiber optical transmission links are limited in transmission distance by accumulation of linear noise from in-line amplifiers and by distortions on the signal from fiber nonlinearity. Phasesensitive amplifiers (PSAs) can alleviate both of these limitations simultaneously. PSAs can be implemented using parametric nonlinearities in either $\chi^{2}$ or $\chi^{3}$ materials and experimental demonstrations of ultra-low noise figure (NF) amplification ${ }^{1}$, all-optical nonlinearity cancellation based on PSA coherent superposition ${ }^{2-4}$ and all-optical phase-regeneration ${ }^{5,6}$ have all been presented. Nonlinearity mitigation based on coherent superposition can also be performed in the digital domain ${ }^{7}$, but then only after full transmission. Using PSAs as in-line amplifiers, nonlinearity mitigation can be performed span-wise.

Multi-span transmission using in-line PSAs has previously only been demonstrated using a PSA operating as a regenerator of binary phase-shift keying (PSK) data ${ }^{6}$. While showing significant improvement in transmission reach, the system was only compatible with BPSK, and the improvement was due to combined phase squeezing and gain saturation, not due to low noise amplification.

Here, we implement a fiber-based PSA with large net gain $(21 \mathrm{~dB})$ operating as an inline amplifier in a loop transmission experiment, and show a three times increase in reach over an erbium-doped fiber amplifier (EDFA) using 10 GBd quadrature phase-shift keying (QPSK) data. The reach increase comes from both the lower NF and the nonlinearity mitigation which allows for a larger accumulated nonlinear phase shift. This represents the first demonstration of an in-line fiber-based PSA in a multi-span long-haul transmission, the first demonstration of span-wise nonlinearity mitigation and the first demonstration of increased reach in both linear and nonlinear transmission regimes by using a low-noise PSA in multi-span transmission.

\section{Experimental setup}

The experimental setup is illustrated in Fig. 1. A signal with $10 \mathrm{GBd}$ QPSK data was combined with a continuous wave (CW) pump using a wavelength division multiplexing (WDM) coupler. Two acousto-optic modulators (AOMs) together with a $3 \mathrm{~dB}$ coupler were used to control the switching of waves in and out of the loop. The signal and pump launched into the loop were attenuated using variable optical attenuators (VOAs) to match the power level after following circulations. Before the in-line amplifier the pump was separated from the signal (and idler) and passed into a pump recovery system for amplification and regeneration $^{8}$. The signal (and idler) was passed through another arm and then combined with the high-power pump before being launched into fiber optical parametric amplifier (FOPA) which was implemented with strained highly nonlinear fiber (HNLF) with in-line isolators ${ }^{9}$. During the first round trip, with only pump and signal present at the input of the FOPA, the FOPA operated as a phase-insensitive amplifier (PIA), generating a conjugated copy of the signal at the idler wavelength. For the following round trips the FOPA operated as a phase-sensitive amplifier (PSA). The gain of the PIA was $16 \mathrm{~dB}$ and the gain of the PSA was $21 \mathrm{~dB}$. The PSA NF was estimated to be about $1.1 \mathrm{~dB}$. Polarization controllers (PCs) were used to align polarizations to maximize the PIA and PSA gain. The signal and idler waves were then separated from the pump and amplified by an EDFA before being passed through an optical processor for delay and amplitude trimming. The pump was attenuated to get $2.5 \mathrm{dBm}$ at the standard single mode fiber (SSMF) input, $P_{\text {in }}$. The transmission link consisted of a dispersion compensating 


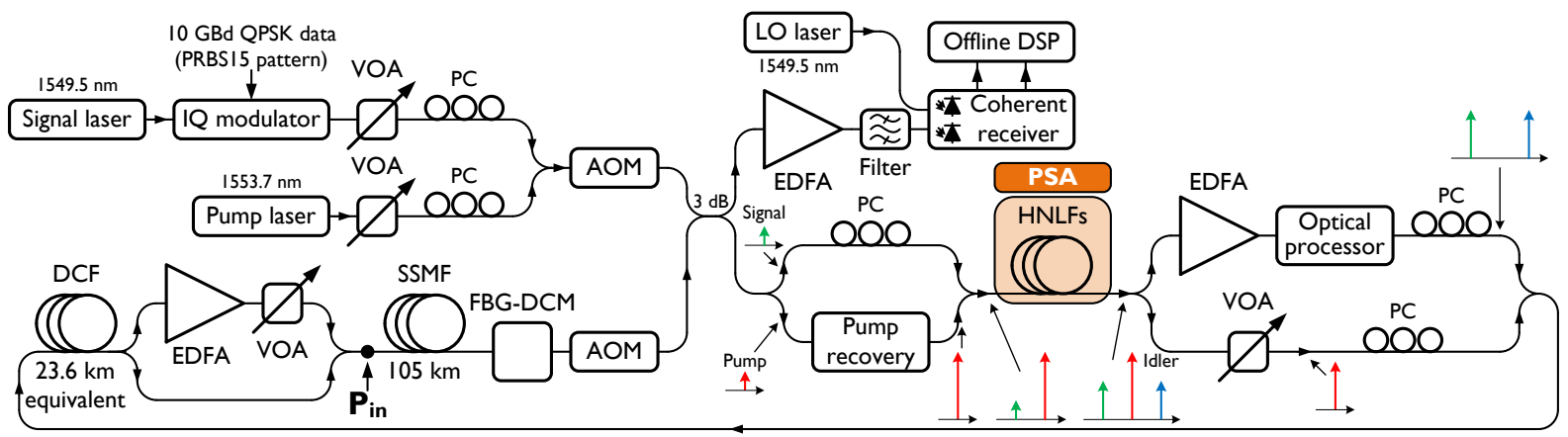

Fig. 1: Experimental setup used for demonstration of multi-span in-line phase-sensitive amplification. Arrows indicate waves during the first round trip for the PSA case. Acronyms are explained in the text.

fiber (DCF) pre-compensation stage equivalent to $23.6 \mathrm{~km}$ SSMF, a signal and idler amplification stage for varying signal power launched into the transmission link, a $105 \mathrm{~km}$ long SSMF transmission fiber, and finally a fiber Bragg-grating dispersion-compensating module (FBG-DCM) for post-compensation. The dispersion map was chosen for efficient mitigation of nonlinearities ${ }^{3}$. The total link loss from the SSMF input to the inline amplifier input was $38 \mathrm{~dB}$. After switching out the signal, it was amplified by an EDFA and detected with a coherent receiver. Standard digital signal processing (DSP) for QPSK was used to recover the signal and then calculate bit error ratio (BER). In the EDFA case the FOPA was substituted with an EDFA with an estimated NF of about $4.0 \mathrm{~dB}$, and a VOA to get $20 \mathrm{~dB}$ net gain. No pump or idler wave was transmitted. Measurements were also done with a phase-insensitive (PI)-FOPA in which case the idler was blocked in the optical processor (OP) and the pump was generated locally in the pump recovery stage.

\section{Results and discussion}

In Fig. 2, we show constellation diagrams of the received signal at $B E R=10^{-3}$ after linear and nonlinear transmission. In the linear transmission regime, EDFA amplification supports four round trips while PSA amplification supports 17. After four round trips, the constellation exhibits much less noise in the PSA case than in the EDFA case. This result is in line with the four times increase in reach that is theoretically expected when using a copier-multiple-PSA link instead of a PIA-only link ${ }^{10}$. We can also compare our result with an EDFA link with two waves carrying the same data, which would only double the reach at the same expense in spectral utilization ${ }^{11}$.

In the nonlinear transmission regime, the reach increase is three times (30 versus 10 round trips). The constellations for PSA amplification are also shown for 10 round trips, and are evidently much

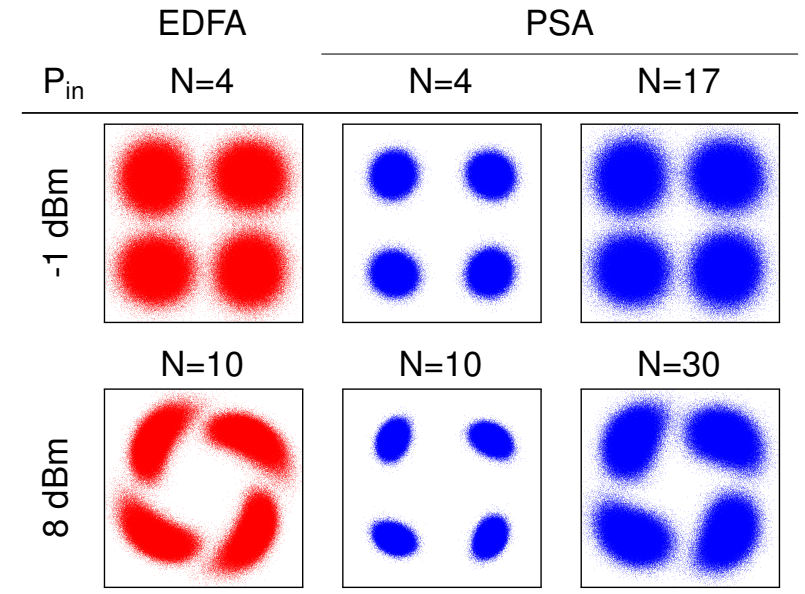

Fig. 2: Constellation diagrams in linear and nonlinear transmission regime giving a BER of $1 \times 10^{-3}$.

less distorted by nonlinear phase rotation than for EDFA amplification. The reach improvement here is believed to be owing primarily to the nonlinearity cancellation performed by the PSA after each span.

BER is plotted versus launch power in Figs. 3 and 4 for EDFA and PSA amplification, respectively. The optimal launch power (assuming $B E R=10^{-3}$ ) is $6 \mathrm{dBm}$ for the EDFA link, giving 11 round trips, i.e. $1050 \mathrm{~km}$ transmission, and $4 \mathrm{dBm}$ for the PSA link, giving 33 round trips, i.e. $3465 \mathrm{~km}$ transmission. While the optimal launch power is $2 \mathrm{~dB}$ lower in the PSA case, the three times longer reach means that the PSA can accommodate approximately twice the accumulated nonlinear phase shift compared to the EDFA (proportional to $P_{\text {in }} \times N$ ).

When operating the PSA amplified link at high launch powers and long transmission distance not only a degradation in BER was seen but it was also increasingly difficult to obtain stable measurement results. The cause for this will require further investigation but it was observed that cycle slips in the DSP play a role as well as pump degradation over increasing distances due to imperfect pump recovery. 


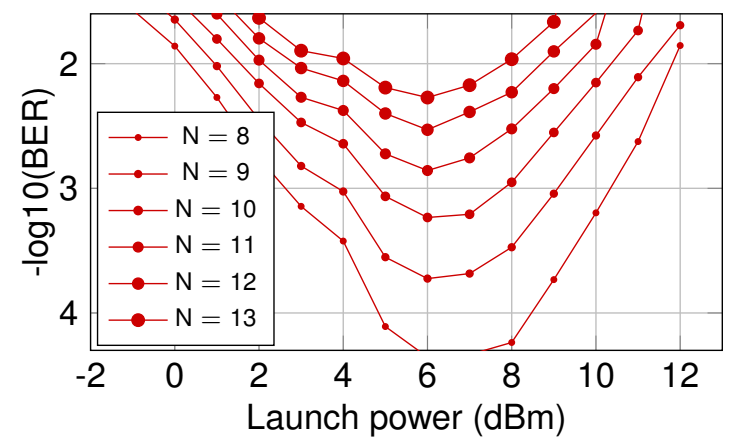

Fig. 3: BER versus launch power for various number of

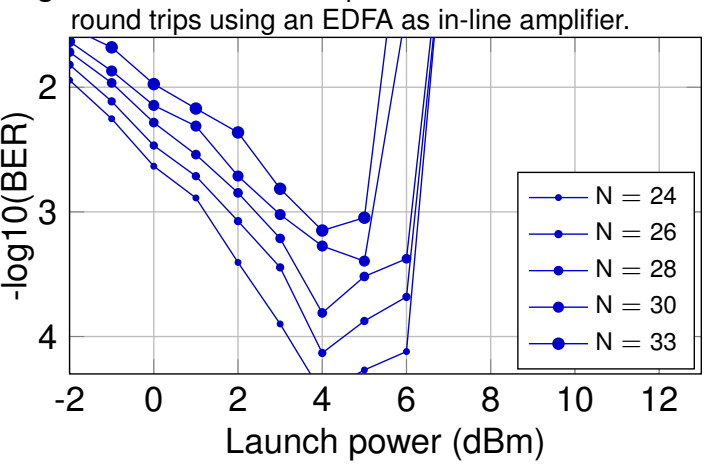

Fig. 4: BER versus launch power for various number of round trips using a PSA as in-line amplifier.

The constellations after transmission at optimal launch powers are shown in Fig. 5. It is readily seen that EDFA amplified transmission is significantly limited by nonlinear distortions. In PSA amplified transmission, it is likely a combination of noise and nonlinearities that limits the reach, as the constellation points are much more symmetric in this case.

The BER versus number of round trips in the various cases studied are shown in Fig. 6, highlighting the significant increase in reach for PSA amplified transmission over EDFA amplified transmission, for low, high, and optimal launch powers. Finally, we note that similar performance as for the EDFA case was obtained using the FOPA in $\mathrm{PI}$-mode. We expect further improvement of the PSA performance for high launch powers is possible with improved pump recovery.

\section{Conclusions}

We have demonstrated the first combined low NF amplification and nonlinearity mitigation using an in-line PSA in a multi-span optical transmission link. Using QPSK, a transmission distance of $3465 \mathrm{~km}$ was possible with the PSA, while using an EDFA the maximum reach was $1050 \mathrm{~km}$.

\section{Acknowledgements}

OFS Denmark are acknowledged for providing HNLFs. This work is supported by the European Research Council under grant agreement ERC-

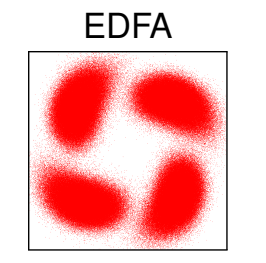

$P_{\text {in }}=6 \mathrm{dBm}, \mathrm{N}=11$

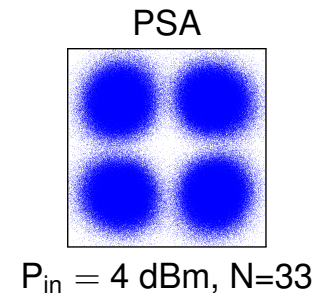

Fig. 5: Constellation diagrams for in-line EDFA and PSA amplification at optimal launch powers and $B E R=1 \times 10^{-3}$.

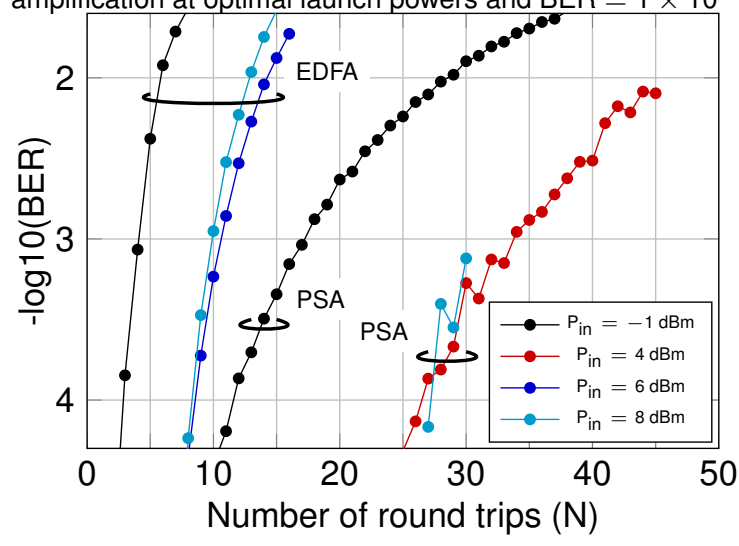

Fig. 6: BER versus number of round trips for EDFA and PSA in-line amplification at $-1 \mathrm{dBm}$ signal launch power (linear transmission), $4 \mathrm{dBm}$ (optimum for PSA), $6 \mathrm{dBm}$ (optimum for

EDFA), and $8 \mathrm{dBm}$ (strongly nonlinear transmission).

2011-AdG - 291618 PSOPA and by the Wallenberg Foundation.

\section{References}

[1] Z. Tong et al., "Towards Ultrasensitive Optical Links Enabled by Low-Noise Phase-Sensitive Amplifiers," Nat. Photon., Vol. 5, no 7, p. 430 (2011).

[2] S.L.I. Olsson et al., "Phase-Sensitive Amplified Optical Link Operating in the Nonlinear Transmission Regime," Proc. ECOC, Th.2.F.1, Amsterdam (2012).

[3] B. Corcoran et al., "Mitigation of Nonlinear Impairments on QPSK Data in Phase-Sensitive Amplified Links," Proc. ECOC, We.3.A.1, London (2013).

[4] S.L.I. Olsson et al., "Linear and Nonlinear Transmission of 16-QAM over 105 km Phase-Sensitive Amplified Link," Proc. OFC, Th1H.3, San Francisco (2014).

[5] R. Slavík et al., "All-Optical Phase and Amplitude Regenerator for Next-Generation Telecommunications Systems," Nat. Photon., Vol. 4, no 10, p. 690 (2010).

[6] T. Umeki et al., "Multi-Span Transmission Using Phase and Amplitude Regeneration in PPLN-based PSA," Opt. Express, Vol. 21, no. 15, p. 18170 (2013).

[7] X. Liu, et al., "Phase-Conjugated Twin Waves for Communication Beyond the Kerr Nonlinearity Limit," Nat. Photon., Vol. 7, no. 7, p. 560 (2013).

[8] S.L.I. Olsson et al., "Injection-Locking Based Pump Recovery for Phase-Sensitive Amplified Links," Opt. Express., Vol. 21, no. 12, p. 14512 (2013).

[9] C. Lundström et al., "Fiber Optic Parametric Amplifier With 10-dB Net Gain Without Pump Dithering," Phot. Technol. Lett., Vol. 25, no. 3, p. 234 (2013).

[10] Z. Tong et al., "Noise Performance of Optical Fiber Transmission Links that Use Non-Degenerate Cascaded Phase-Sensitive Amplifiers," Opt. Express, Vol. 18, no. 15 , p. 15426 (2010).

[11] B. Corcoran et al., "Phase-Sensitive Optical PreAmplifier Implemented in an 80km DQPSK Link,", Proc. OFC, PDP5A.4, Los Angeles (2012). 